\title{
Rip brane cosmology from a viscous holographic dark fluid
}

\author{
I. Brevik ${ }^{1}$ \\ ${ }^{1}$ Department of Energy and Process Engineering, \\ Norwegian University of Science and Technology, N-7491 Trondheim, Norway \\ A. V. Timoshkin ${ }^{2,3}$ \\ ${ }^{2}$ Tomsk State Pedagogical University, \\ Kievskaja Street, 60, 634061 Tomsk, Russia and \\ ${ }^{3}$ Tomsk State University of Control Systems and Radio Electronics, \\ Lenin Avenue, 36, 634050 Tomsk, Russia
}

(Dated: April 1, 2020)

\begin{abstract}
This article is devoted to the application of the holographic principle to describe Rip brane cosmological models in the presence of a bulk viscosity. We make use of the generalized infraredcutoff holographic dark energy, introduced by Nojiri and Odintsov. We consider various examples: Rip brane cosmology corresponding to the Little Rip case, asymptotic de Sitter theory, and the so-called Big Freeze theory leading to a singularity. Analytical expressions for infrared cutoffs, as well as the particle and the future horizons at the brane, are obtained. The equations for energy conservation on the brane within the holographic theory are obtained in each case. The correspondence between viscous cosmology and holographic cosmology on the brane is shown.
\end{abstract}


Keywords: Viscous cosmology; rip brane cosmology; holographic dark fluid Mathematics Subject Classification 2010: 83F05

\section{INTRODUCTION}

An interesting approach to the explanation of the accelerated expansion of the Universe in terms of the dark energy concept, is the application of the so-called holographic principle [1]. The generalized cutoff holographic dark energy model was proposed by Nojiri and Odintsov [2, 3]. Further investigations with application to holographic dark energy in the late-time cosmology were presented in several papers [4 13]. The holographic principle can be realized also in the early universe, obtaining inflation [14], and a cosmological bounce [15], having a holographic origin. It has been shown that the application of the theory of holographic dark energy in the late-time universe is consistent with astronomical observations [16 20]. General reviews of different approaches to dark energy are given in Refs. [21] and [22].

In the late-time universe there is a possibility of forming a brane cosmological model [23, 24]. The brane scenario is one among several approaches for how to solve the singularity problem [25]. Several dark energy models on the brane, with various scenarios of evolution, have been investigated in Refs. [26-29].

The present article is devoted to an application of the principle of a generalized holographic cutoff [3] in order to describe the brane dark energy models. We will start from the basic brane models proposed in [26, 27], and investigate the evolution of the cosmic fluid assuming that the fluid possesses a bulk viscosity. The most convenient formalism for constructing the (scalar or fluid) dark energy is to use the equation of state (EoS). The evolution of the universe then depends on the choice of the EoS. In accordance with usual $4 \mathrm{~d}$ Friedman-Robertson-Walker (FRW) cosmology, we will consider the Little Rip behavior, the asymptotic de Sitter evolution, and the Big Freeze singularity. The viscous contribution is effectively taken into account via the corresponding choice of an inhomogeneous dark fluid. We will apply the holographic principle to the late-time epoch, associating the choice made by Nojiri and Odintsov for the infrared cutoff with future events and particle horizons, and we will derive the corresponding forms of the energy conservation law. Thereby we establish the equivalence between our viscous fluid models on the brane and the holographic models on the brane. 
In the next section we recall the main points of the holographic principle applied to the description of the universe. The third section is devoted to the study of cosmological models on the brane, taking into account the viscosity property of the dark fluid, showing the equivalence between viscous fluid cosmology and holographic fluid cosmology on the brane. The final section summarizes the obtained results.

\section{THE HOLOGRAPHIC DARK ENERGY}

In this section we give the main points of the holographic principle, following the terminology proposed by Li [17] (string theory uses a different terminology [30]). The holographic description of dark energy, based on the holographic principle, states that the cutoff radius of the horizon may be related to the infrared cutoff. The generalized holographic energy density was proposed in Ref. [2], where the infrared cut-off was identified with a combination of FRW universe parameters: the Hubble parameter, the particle and the future event horizons, the cosmological constant, and the finite life time of the universe.

In general the holographic energy density is proportional to the inverse squared infrared cutoff $L_{I R}[3]$

$$
\rho=\frac{3 c^{2}}{k^{2} L_{I R}^{2}},
$$

where $k^{2}=8 \pi G$ is Einsteins gravitational constant and $c$ is a dimensionless parameter (thus not the light velocity).

In order to apply equation (11) in a cosmological framework we consider a homogeneous and isotropic FRW flat universe

$$
d s^{2}=-d t^{2}+a^{2}(t) \sum_{i=1,2,3}\left(d x^{i}\right)^{2},
$$

where $a$ is the scale factor. The first FRW equation has the form

$$
H^{2}=\frac{k^{2}}{3} \rho,
$$

where $\rho$ is the holographic energy density. Then,

$$
H=\frac{c}{L_{I R}} .
$$

Actually there is no definite prediction for the choice of the infrared cutoff at present; one can choose the particle horizon, or the future event horizon, whose definitions are given in 
Ref. (3),

$$
L_{p} \equiv a(t) \int_{0}^{t} \frac{d t^{\prime}}{a\left(t^{\prime}\right)}, \quad L_{f}(t) \equiv a(t) \int_{t}^{\infty} \frac{d t^{\prime}}{a\left(t^{\prime}\right)} .
$$

In the general case one can construct an infrared cutoff as a combination of these quantities

and their derivatives [2]. Using the infrared radius in this form, one can apply the holographic principle to describe the late-time universe.

Further, we will assume that the viscous fluid, responsible for the accelerated expansion of the universe, is related to the holographic energy density.

\section{HOLOGRAPHIC DESCRIPTION OF RIP BRANE FRW VISCOUS COS- MOLOGY}

One of several ways to solve the singularity problem is to apply the brane world scenario. According to the simplest version the space-time is homogeneous and isotropic along the three spatial dimensions, this being our 4-dimension universe, while there is a thin wall

and constant spatial curvature when viewed from a 5-dimensional space-time [31 33]. The Friedmann equation on the brane turns out to be different from the standard cosmology equation. The Hubble parameter has the following form

$$
H^{2}=\frac{k^{2}}{3} \rho\left(1+\frac{\rho}{2 \lambda}\right)
$$

where $H=\dot{a} / a$ and $\lambda$ is the brane tension. If the inequality $\rho \ll|\lambda|$ holds, then Eq. (6) differs insignificantly from the first FRW Eq. (33). Thus, one can assume that in our epoch $\rho / 2 \lambda \ll 1$, and there will be no significant difference between the brane model and conventional FRW cosmology.

Next, we consider examples of holographic dark energy models on the brane, corresponding to the Little Rip model, the de Sitter asymptotic theory, and the so-called type III cosmological singularity model. We take into account the bulk viscosity of the fluid throughout. We will suppose, for simplicity, that the universe consists of dark energy only. We assume that this universe satisfies an inhomogeneous equation of state (EoS) in flat FRW space-time [34, 35],

$$
p=\omega(\rho, t) \rho-3 H \zeta(H, t),
$$

where $\omega(\rho, t)$ is the thermodynamic parameter and $\zeta(H, t)$ the bulk viscosity, depending on the Hubble parameter and on the time $t$. According to conventional thermodynamic, it is 
natural to assume that $\zeta>0$. We will take the following form for the thermodynamic EoS parameter [34, 35]

$$
\omega(\rho, t)=\omega_{1}(t)\left(A_{0} \rho^{\alpha-1}-1\right)
$$

where $A_{0} \neq 0$ and $\alpha \geq 1$ are constants. We choose the bulk viscosity as [34, 35]

$$
\zeta(H, t)=\zeta_{1}(t)(3 H)^{n}
$$

with $n>0$.

As mentioned, we assume that the universe consists of a one-component viscous fluid, whose energy conservation equation is

$$
\dot{\rho}+3 H(\rho+p)=0 .
$$

Further, we will investigate the future evolution of the universe on the brane, in the holo-

graphic picture. For this we will apply the holographic principle on the brane for different cases, and for different forms for the bulk viscosity term, in order to obtain the appropriate energy conservation law in each case.

\section{A. Little Rip case}

Let us consider a nonsingular brane Little Rip model, in which the scale factor is given as [26, 27]

$$
a(t)=a_{0} \exp \left[\frac{\lambda}{3 \alpha^{2}} \cosh \left(\sqrt{\frac{3 \alpha^{3}}{2 \lambda}} t\right)\right], \quad \lambda>0 .
$$

Here it is natural to associate $t=0$ with the present time; then $a_{0} \exp \left(\lambda / 3 \alpha^{2}\right)$ corresponds to the present time scale factor. Various scenarios of the Little Rip were discussed in Refs. [36, 37].

We now adopt a constant value for the EoS parameter, $\omega(\rho, t)=\omega_{0}$, and assume a linear dependence of the bulk viscosity on the Hubble parameter, $\zeta(H, t)=3 \tau H$, with $\tau$ a positive dimensional constant. Then taking into account Eq. ([6), we obtain the EoS (17) in the form

$$
p=\omega_{0}\left(\sqrt{\lambda^{2}+6 \frac{\lambda}{k^{2}} H^{2}}-\lambda\right)-9 \tau H^{2} .
$$


Let us choose to consider the holographic principle on the brane in terms of the future horizon $L_{f},[5]$. After some calculation we obtain

$$
L_{f}=\sqrt{\frac{\lambda}{6 \alpha^{3}}}\left\{\begin{array}{l}
\exp \left[\frac{\lambda}{3 \alpha^{2}}\left(\cosh \sqrt{\frac{3 \alpha^{3}}{2 \lambda}} t+\frac{1}{\sqrt{2}}\right)\right] E_{1}\left[\cosh \sqrt{\frac{3 \alpha^{3}}{2 \lambda}} t+\frac{\lambda}{3 \sqrt{2} \alpha^{2}}\right]+ \\
+\exp \left[\frac{\lambda}{3 \alpha^{2}}\left(\cosh \sqrt{\frac{3 \alpha^{3}}{2 \lambda}} t-\frac{1}{\sqrt{2}}\right)\right] E_{1}\left[\cosh \sqrt{\frac{3 \alpha^{3}}{2 \lambda}} t-\frac{\lambda}{3 \sqrt{2} \alpha^{2}}\right],
\end{array}\right\}
$$

where $E_{1}(x)$ is the integral exponential function.

The expressions for the Hubble parameter in terms of the future event horizon $L_{f}$ and its time derivatives are [3]

$$
H=\frac{\dot{L}_{f}+1}{L_{f}}, \quad \dot{H}=\frac{\ddot{L}_{f}}{L_{f}}-\frac{\dot{L}_{f}^{2}}{L_{f}^{2}}-\frac{\dot{L}_{f}}{L_{f}^{2}} .
$$

Thus by using Eqs. (12) and (14) the energy conservation equation (10) takes the following form in the holographic language,

$$
\begin{gathered}
\frac{2 \lambda}{k} \frac{\ddot{L}_{f}-\frac{\dot{L}_{f}^{2}+\dot{L}_{f}}{L_{f}}}{\sqrt{\left(\lambda k L_{f}\right)^{2}+6 \lambda\left(\dot{L}_{f}+1\right)^{2}}}-9 \tau\left(\frac{\dot{L}_{f}+1}{L_{f}}\right)^{2}+ \\
+\left(\omega_{0}+1\right)\left[-\lambda+\frac{1}{k L_{f}} \sqrt{\left(\lambda k L_{f}\right)^{2}+6 \lambda\left(\dot{L}_{f}+1\right)^{2}}\right]=0 .
\end{gathered}
$$

This is thus he realization of the holographic principle on the brane for a viscous fluid within the Little Rip model.

\section{B. Asymptotic de Sitter regime}

In this case the brane has a negative tension $(\lambda<0)$, so that the universe expands in a quasi-de Sitter regime. The scale factor increases with time as [26, 27]

$$
a(t)=a_{0} \exp \left\{-\frac{\beta^{2}}{2}\left[\frac{1}{\cos \eta_{0}}-\sqrt{1+\left(\tan \eta_{0}+\frac{\alpha}{\beta} t\right)^{2}}\right]\right\} .
$$

Here $\beta^{2}=\frac{2|\lambda|}{3 \alpha^{2}}$ is a dimensionless parameter and $\eta_{0}=\sqrt{\frac{3}{2 \lambda}} \alpha^{2} t_{0}$, where $t_{0}$ is the present time.

Let us study the case of constant bulk viscosity, $\zeta(H, t)=\zeta_{0}>0$, and assume that the thermodynamic parameter $\omega(\rho, t)$ is a linear function of the energy density,

$$
\omega(\rho, t)=A_{0} \rho-1
$$


The the EoS for the viscous fluid (17) takes the form

$$
p=\left(2 A_{0} \lambda+1\right)\left(\lambda-\sqrt{\lambda^{2}+\frac{6 \lambda}{k^{2}} H^{2}}\right)+3 H\left(\frac{2 A_{0} \lambda}{k^{2}} H+\xi_{0}\right) .
$$

The holographic description of a viscous dark fluid on the brane, in terms of the future event horizon $L_{f}$, Eq. (15), then becomes

$$
L_{f}=\frac{\beta}{\alpha}\left\{\begin{array}{l}
\frac{2}{\beta^{2}}-\left(\frac{\alpha}{\beta} t+\tan \eta_{0}\right)+\sqrt{1+\left(\frac{\alpha}{\beta} t+\tan \eta_{0}\right)^{2}}+ \\
+\frac{\beta^{2}}{4} \exp \left[\frac{\beta^{2}}{2} \sqrt{1+\left(\frac{\alpha}{\beta} t+\tan \eta_{0}\right)^{2}}\right] E_{i}\left[-\frac{\beta^{2}}{2} \sqrt{1+\left(\frac{\alpha}{\beta} t+\tan \eta_{0}\right)^{2}}\right]
\end{array}\right\},
$$

where $E_{i}$ is an exponential integral function.

Now using Eqs. (14) and (18) we can rewrite the continuity equation for the energy in a holographic language,

$$
\begin{gathered}
\frac{\ddot{L}_{f}-\frac{\dot{L}_{f}}{L_{f}}\left(\dot{L}_{f}+1\right)}{k \sqrt{\left(\lambda k L_{f}\right)^{2}+6 \lambda\left(\dot{L}_{f}+1\right)^{2}}}-\frac{3 \xi_{0}}{2 \lambda} \frac{\dot{L}_{f}+1}{L_{f}}+ \\
+A_{0}\left[\lambda-\frac{1}{k L_{f}} \sqrt{\left(\lambda k L_{f}\right)^{2}+6 \lambda\left(\dot{L}_{f}+1\right)^{2}}+\frac{3}{\left(k L_{f}\right)^{2}}\left(\dot{L}_{f}+1\right)^{2}\right]=0 .
\end{gathered}
$$

Thus, we have constructed a holographic model on the brane, taking into account the fluid viscosity in the de Sitter asymptotic regime.

\section{Big Freeze singularity cosmology}

Let us assume that the brane tension is positive, $\lambda>0$. There are then two Big Freeze singularities: one in the past $(t \rightarrow-\infty)$ and the other in the future $(t \rightarrow \infty)$. The universe begins its existence at $t_{i n}=-\frac{1}{\alpha} \sqrt{\frac{2 \lambda}{3 \alpha}}$ and ends at $t_{f}=+\frac{1}{\alpha} \sqrt{\frac{2 \lambda}{3 \alpha}}$.

The scale factor is [26, 27]

$$
a(t)=a_{f} \exp \left[-\frac{\lambda}{3 \alpha^{2}}\left(1-\frac{3 \alpha^{3} t^{2}}{2 \lambda}\right)^{1 / 2}\right]
$$

where $a_{f}$ is the final scale factor. This expression shows that the universe contracts in the time interval $t_{i n}<t<0$ and then expands.

In this model we will assume the thermodynamic parameter $\omega(\rho, t)$ to be a linear function

of the energy density (17), and also assume the bulk viscosity $\zeta(H, t)$ to be a linear function 
of $H$. Then, following (17, we write the EoS as

$$
p=2 A_{0} \lambda^{2}-\left(2 A_{0} \lambda+1\right) \sqrt{\lambda^{2}+6 \frac{\lambda}{k^{2}} H^{2}}+3\left(\frac{2 A_{0} \lambda}{k^{2}}-3 \tau\right) H^{2},
$$

and calculate the particle horizon, following (5), as

$$
L_{p}=2 \sqrt{\frac{\pi}{\alpha}} \exp \left[\frac{\lambda}{3 \alpha^{2}}\left(1-\sqrt{1-\frac{3 \alpha^{3}}{2 \lambda} t^{2}}\right)\right] \operatorname{erf}\left(\frac{1}{2} \sqrt{\alpha} t\right),
$$

where erf $\left(\frac{1}{2} \sqrt{\alpha} t\right)$ is a probability integral.

Substituting expressions for the Hubble parameter in terms of the particle horizon $L_{p}$ and its time derivatives [3], we get

$$
H=\frac{\dot{L}_{p}-1}{L_{p}}, \quad \dot{H}=\frac{\ddot{L}_{p}}{L_{p}}-\frac{\dot{L}_{p}^{2}}{L_{p}^{2}}+\frac{\dot{L}_{p}}{L_{p}^{2}} .
$$

Moreover, substituting the EoS in the form (22) in the continuity equation for energy (10), we get in this cosmological model

$$
\begin{gathered}
\frac{\ddot{L}_{p}-\frac{\dot{L}_{p}}{L_{p}}\left(\dot{L}_{p}-1\right)}{k \sqrt{\left(\lambda k L_{p}\right)^{2}+6 \lambda\left(\dot{L}_{p}-1\right)^{2}}}-3\left(\frac{A_{0}}{k^{2}}-\frac{3 \tau}{2 \lambda}\right)\left(\frac{\dot{L}_{p}-1}{L_{p}}\right)^{2}+ \\
+A_{0}\left[\lambda-\frac{1}{k L_{p}} \sqrt{\left(\lambda k L_{p}\right)^{2}+6 \lambda\left(\dot{L}_{p}-1\right)^{2}}\right]=0 .
\end{gathered}
$$

This shows the behavior of the Big Freeze singularity within the viscous holographic model.

\section{CONCLUSION}

In the present work we have applied the holographic principle to late-time cosmology on the brane, considering various cosmological models for the rip brane holographic scenario. The mathematical tool for description was the inhomogeneous equation of state for a onecomponent dark fluid having a bulk viscosity. For each of the considered models, using analytical calculations, we arrived at expressions for the infrared cutoff in the form of a particle horizon, or an event horizon. We reformulated the continuity equation for the energy density of the viscous fluid in the holographic language. Thereby, the equivalence between the description of cosmology on the brane using a viscous fluid, and the holographic description using infrared cutoff as proposed by Nojiri and Odintsov [2, 3], was shown. This 
theory can be extended to the case two coupled fluids with calculations similar to those given here.

A natural question is whether the predictions of the holographic theory agree with astronomical observations. An analysis of the correspondence between theoretical models of holographic dark energy on the brane and astronomical observations was carried out in [38], whereby it became possible to determine the upper limit of the ratio between the present energy density and tension on the brane. In that article the relationships between apparent magnitudes and redshifts for distant supernovae Ia, Hubble parameters for various redhifts, and baryon acoustic oscillations were obtained. For a wide range of parameters, the observational data were well described.

[1] M. Li, A Model of Holographic Dark Energy. Phys. Lett. B 603, 1 (2004) hep-th/0403127.

[2] S. Nojiri and S. D. Odintsov, Unifying phantom inflation with late-time acceleration: scalar phantom-non-phantom transition model and generalized holographic dark energy. Gen. Rel. Grav. 38, 1285 (2006) hep-th/0506212].

[3] S. Nojiri and S. D. Odintsov. Covariant generalized holographic dark energy and accelerating universe. Eur. Phys. J. C 77, 528 (2017), arXiv:1703.06372 [hep-th]].

[4] S. Wang, Y. Wang and M. Li, Holographic Dark Energy. Phys. Rept. 696, 1 (2017) [1612.00345 [astro-ph.CO]].

[5] R. Horvat, Holography and Variable Cosmological Constant. Phys. Rev. D 70, 087301 (2004) astro-ph/0404204.

[6] Q. G. Huang and M. Li, The Holographic Dark Energy in a Non-flat Universe. JCAP 0408, 013 (2004) astro-ph/0404229.

[7] D. Pavon andW. Zimdahl, Holographic dark energy and cosmic coincidence. Phys. Lett. B 628, 206 (2005) gr-qc/0505020.

[8] B. Wang, Y. g. Gong and E. Abdalla, Transition of the dark energy equation of state in an interacting holographic dark energy model. Phys. Lett. B 624, 141 (2005) hep-th/0506069.

[9] M. R. Setare and E. N. Saridakis, Non-minimally coupled canonical, phantom and quintom models of holographic dark energy. Phys. Lett. B 671, 331 (2009) [0810.0645 [hep-th]]. 
[10] K. Enqvist and M. S. Sloth, A CMB/Dark Energy Cosmic Duality. Phys. Rev. Lett. 93, 221302 (2004) hep-th/0406019.

[11] X. Zhang, Statefinder diagnostic for holographic dark energy model. Int. J. Mod. Phys. D 14, 1597 (2005) astro-ph/0504586].

[12] B. Guberina, R. Horvat and H. Stefancic, Hint for Quintessence-like Scalars from Holographic Dark Energy. JCAP 0505, 001 (2005) astro-ph/0503495].

[13] E. Elizalde, S. Nojiri, S. D. Odintsov and P. Wang, Dark Energy:Vacuum Fluctuations, the Effective Phantom Phase, and Holography. Phys. Rev. D 71, 103504 (2005) [hep-h/0502082].

[14] S. Nojiri, S. D. Odintsov and E. N. Saridakis, Holographic inflation. Phys. Lett. B 797, 134829 (2019) arXiv:1904.01345v1 [gr-qc]].

[15] S. Nojiri, S. D. Odintsov and E. N. Saridakis, Holographic bounce. (2019) arXiv:1908.00389 $[\mathrm{gr}-\mathrm{qc}]]$.

[16] X. Zhang and F. Q. Wu, Constraints on holographic dark energy from type Ia supernova observations. Phys. Rev. D 72, 043524 (2005) [astro-ph/0506310].

[17] M. Li, X. D. Li, S. Wang and X. Zhang, Holographic dark energy models: A comparison from the latest observational data. JCAP 0906, 036 (2009) [0904.0928 [astro-ph.CO]].

[18] J. Lu, E. N. Saridakis, M. R. Setare and L. Xu, Observational constraints on holographic dark energy with varying gravitational constant. JCAP 1003, 031 (2010) [0912.0923 [astro-ph.CO]].

[19] Q. G. Huang and Y. G. Gong, Supernova Constraints on a holographic dark energy model. JCAP 0408, 006 (2004) astro-ph/0403590].

[20] B. Wang, E. Abdalla and R. K. Su, Constraints on dark energy from holography. Phys. Lett. B 611, 21 (2005) hep-th/0404057.

[21] K. Bamba, S. Capozziello, S. Nojiri and S.D. Odintsov, Dark energy cosmology: the equivalent description via different theoretical models and cosmography tests. Astrophys. Space Sci. 342, 155 (2012) [arXiv:1205.3421 [gr-qc]].

[22] S. Nojiri, S.D. Odintsov and V.K. Oikonomou, Modified Gravity Theories on a Nutshell: Inflation, Bounce and Late-time Evolution. Phys. Rept. 692, 1 (2017) arXiv:1705.11098 [gr$\mathrm{qc}]]$.

[23] L. Randall and R. Sundrum, Large Mass Hierarchy from a Small Extra Dimension. Phys. Rev. Lett. 83, 3370 (1999).

[24] L. Randall and R. Sundrum, An alternative to Compactification. Phys. Rev. Lett. 83, 4690 
(1999).

[25] X. Zhang, Avoiding the cosmic doomsday in the holographic dark energy model. Phys. Lett. B 683, 81 (2010).

[26] A. V. Astashenok and S. D. Odintsov, Confronting dark energy models mimicking CDM epoch with observational constraints: future cosmological perturbations decay or future Rip? Phys. Lett. B 718, 1194 (2013), arXiv:1211.1888.

[27] A. V. Astashenok, E. Elizalde, S. D. Odintsov and A. V. Yurov, Equation-of-state formalism for dark energy models on the brane and the future of brane universes. Eur. Phys. J. C 72, 2260 (2012), [arXiv: 1206.2192v1[gr-qc]].

[28] A. V. Astashenok, S. Nojiri, S. D. Odintsov and A. V. Yurov, Phantom Cosmology without Big Rip Singularity. Phys. Lett. B 709, 396 (2012), arXiv:1201.4056v2[gr-qc]].

[29] A. V. Astashenok, S. Nojiri, S. D. Odintsov and R. J. Scherrer, Scalar dark energy models mimicking $\Lambda$ CDM with arbitrary future evolution. Phys. Lett. B 713, 145 (2012), arXiv:1203.1976v2[gr-qc]].

[30] J. Maldacena, Non-Gaussian features of primordial fluctuations in single field inflationary models. JHEP 0305, 013 (2003).

[31] V. Sahni, Yu. Shtanov, Cosmic Acceleration and Extra Dimension, In Problems of Modern Cosmology. (2008) arXiv:0811.3839 [astro-ph]].

[32] D. Langlois, Brane Cosmology. Prog. Theor. Phys. Suppl. 148, 181 (2003).

[33] A. Lue and G. D. Starkman, From Quantum to Cosmos. Phys.Rev. D 70, 101501 (2004).

[34] S. Capozziello, V.F. Cardone, E. Elizalde, S. Nojiri and S.D. Odintsov, Observational constraints on dark energy with generalized equations of state. Phys. Rev. D 73, 043512 (2006).

[35] E. Elizalde, V. V. Obukhov and A. V. Timoshkin, Inhomogeneous viscous dark fluid coupled with dark matter in the FRW universe. Mod. Phys. Lett. A 29, 1450132 (2014).

[36] I. Brevik, E. Elizalde, S. Nojiri and S.D. Odintsov, Viscous Little Rip Cosmology. Phys. Rev. D 84, 103508 (2011), arXiv:1107.4642 [hep-th]].

[37] P.H. Frampton, K.J. Ludwick, S. Nojiri, S.D. Odintsov and R.J. Scherrer, Models for Little Rip Dark Energy. Phys. Lett. B 708, 204 (2012).

[38] A. V. Astashenok, A. S. Tepliakov, Some models of holographic dark energy on the RandallSundrum brane and observational data. arXiv:1911.01422 v1[gr-qc] (2019). 